International Journal of Neural Systems, Vol. 0, No. 0 (April, 2000) 00-00

(C) World Scientific Publishing Company

\title{
DISTRIBUTED SENSOR NETWORKS: A CELLULAR NONLINEAR NETWORK PERSPECTIVE
}

\author{
MARTIN HAENGGI \\ Dept. of Electrical Engineering \\ University of Notre Dame \\ Notre Dame, IN 46556, USA \\ E-mail: mhaenggi@nd.edu \\ Received (to be inserted \\ Revised by Publisher)
}

\begin{abstract}
Large-scale networks of integrated wireless sensors become increasingly tractable. Advances in hardware technology and engineering design have led to dramatic reductions in size, power consumption, and cost for digital circuitry, and wireless communications. Networking, self-organization, and distributed operation are crucial ingredients to harness the sensing, computing, and computational capabilities of the nodes into a complete system. This article shows that those networks can be considered as cellular nonlinear networks (CNNs), and that their analysis and design may greatly benefit from the rich theoretical results available for $\mathrm{CNNs}$.
\end{abstract}

\section{Introduction}

Pervasive micro-sensing and -actuation may revolutionize the way we understand and manage complex physical systems. The capabilities for detailed physical monitoring and manipulation offer enormous opportunities for almost every scientific discipline. Networks of such devices, so-called distributed sensor networks (DSNs), provide an embedded processing platform with exciting capabilities ${ }^{1,2,3}$. Applications include surveillance in inhospitable environments such as remote geographic regions or toxic locations, sensing and maintenance in large industrical plants, planetary exploration, seismic activity detection, medical sensing, micro-surgery, military surveillance and combat, fingertip accelerometer virtual keyboards, and smart office spaces.

In practically all such applications, key requirements include scalability, robustness with respect to various disturbances and uncertainties, evolvability through autonomous reconfiguration and optimal redistribution of resources ${ }^{4}$.
Wireless connectivity is crucial, since for most envisioned applications, the environment being monitored does not have installed infrastructure for either communications or energy supply. Therefore untethered nodes must rely on small local energy sources and wireless communication channels. Due to energy and interference considerations, the transmission range of a node will only include some of its nearest neighbors (see Fig. 1), and since computation is substantially cheaper than transmission, control and resource allocation algorithms run locally on every node in a distributed fashion. With these properties - a distributed system of locally connected nodes - an DSN can be clearly viewed at as a cellular nonlinear network (CNN); the formal definition will be given in Section 3 .

From a networking point of view, DSNs are certainly relatives of wireless ad hoc networks ${ }^{5,6,7}$, sharing a number of challenges such as energy constraints and routing. On the other hand, general ad hoc networks most likely induce traffic patterns different 
from sensor networks, have other lifetime requirements, and are often considered to consist of mobile nodes $^{8,9}$. In DNSs, most nodes are static; however, the network of basic sensor nodes may be overlaid by more powerful mobile sensors (robots) that can, guided by the basic sensors, move to interesting areas or even track intruders in the case of military applications.

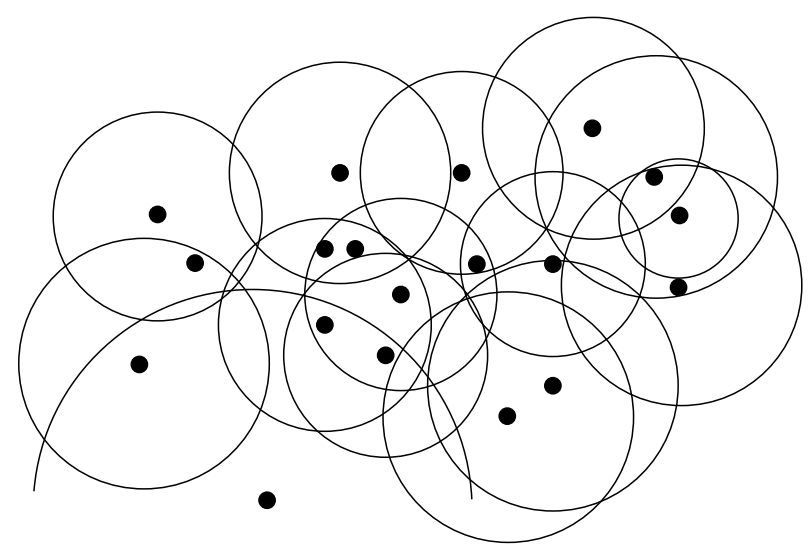

Fig. 1. A DSN with randomly distributed nodes and local connectivity (the circles indicate transmission ranges at a given transmit power level).

\section{Challenges in Distributed Sensor Networks}

In this Section, we will introduce some of the challenges that DSN designers face.

Network nodes are equipped with wireless transmitters and receivers using antennas which may be omnidirectional (isotropic radiation), highly directional (point-to-point), possibly steerable, or some combination thereof. At a given point in time, depending on the nodes' positions and their transmitter and receiver coverage patterns, transmission power levels and co-channel interference levels, a wireless connectivity in the form of a random, multi-hop graph or "ad hoc" network exists between the nodes. This ad hoc topology may change with time as the nodes move or adjust their transmission and reception parameters.

DNSs have several salient characteristics:

- Dynamic topologies. Despite the static nature of the nodes, the network topology may change due to changes in transmit powers or due to random fluctuations in the wireless links. A connection between two nodes usually uses several intermediate nodes as relays (multi-hop connection).

- Bandwidth-constrained, variable capacity wireless links.

- Energy-constrained operation ${ }^{10}$. Since energy is not renewable, every computation and every transmitted bit is costly in terms of network lifetime.

- Limited physical security. Individual nodes may easily be removed or destroyed.

These properties pose formidable challenges to DSN implementations, which will be elaborated on in the following Subsections.

- Energy-efficiency.

- Synchronization and localization. Synchronization is a prerequisite for efficient operation of the network, and without localization, the sensed data is not useful.

- Routing. How can a path be found from one node to a possibly distant destination node if the topology is dynamic, individual nodes are not reliable and only nearest neighbors can be reached directly?

- Channel access. The nodes share the wireless channel as a common communication medium. How can collisions (occurring when two nearby nodes transmit simultenously) be minimized?

- Propagation of information. How can it be guaranteed that sensor data is delivered in a timely manner? In networking terminology, this is the so-called quality of service (QoS) problem.

\subsection{Power Supply}

The most difficult constraints in the design of DSNs are those regarding the minimum energy consumption necessary to drive the circuits and possible micro-electro-mechanical devices (MEMS) ${ }^{11,7,10}$. The energy problem is aggravated if actuators are present that may be substantially more powerhungry than the sensors. When miniaturizing the node, the energy density of the power supply is the primary issue. Current technology yields batteries with approx. $1 \mathrm{~J} / \mathrm{mm}^{3}$ of energy, while capacitors can achieve as much as $1 \mathrm{~mJ} / \mathrm{mm}^{3}$. 
If a node were designed to have a relatively short lifespan, such as a device built to transmit data only a few times, a battery would be a logical solution. However, for nodes that can generate sensor readings for long periods of time, a charging method for the supply must be utilized. Currently, research groups investigate the use of solar cells to charge capacitors with photocurrents from the ambient light sources. Solar flux can yield power densities of approximately $1 \mathrm{~mW} / \mathrm{mm}^{2}$. The energy efficiency of a solar cell ranges from 10-30\% in current technologies, giving $300 \mu \mathrm{W}$ in full sunlight in the best-case scenario for a $1 \mathrm{~mm}^{2}$ solar cell operating at $1 \mathrm{~V}$. Series-stacked solar cells will need to be utilized in order to provide appropriate voltages.

Sensor acquisition can be achieved at 1nJ/sample, and modern processors can perform computations as low as $1 \mathrm{~nJ} /$ instruction. For wireless communications, the primary candidate technologies are based on RF and optical transmission techniques. Each technique has its advantages and disadvantages. RF presents a problem because the nodes may offer very limited space for for antennas, thereby demanding very short-wavelength (i.e., high-frequency) transmission. Communication in that regime is not currently compatible with low power operation. Current RF transmission techniques (e.g., Bluetooth ${ }^{12}$ ) consum about 100nJ/bit for a distance of $10-100 \mathrm{~m}$, making communication very expensive compared to acquisition and processing. An attractive alternative is to employ free-space optical transmission. If a line-of-sight path is available, a well-designed free-space optical link requires significantly lower energy than its RF counterpart, currently about $1 \mathrm{~nJ} /$ bit. The reasons for this power advantage are that optical transceivers require only simple baseband analog and digital circuitry and no modulators, active filters, and demodulators. The extremely short wavelength of visible light makes it possible for a millimeter-scale device to emit a narrow beam, corresponding to an antenna gain of roughly 7 orders of magnitude compared to an isotropic radiator.

In DSNs, where sensor sampling, processing, data transmission, and possibly actuation is involved, the trade-off between these tasks plays an important role in power usage. Given a finite amount of energy, balancing these parameters depending on the application will be the focus of the design process of DSNs.

\subsection{Routing and clustering}

Numerous routing algorithms have been proposed and studied for ad hoc networks ${ }^{13,5}$, but most of them suffer from the drawbacks that they were not specifically developed for DSNs, that they require globally unique addresses, and that entire routes from end-node to end-node have to be discovered and continuously updated. The so-called directed diffusion $^{14}$ algorithm takes a different approach by letting messages pass from node to node, without specifying an exact destination node but rather a geographic area or even just a request for certain information. Nodes only have to be aware of their nearest neighbors; this guarantees that a node that has the desired information can reply via the reverse path. Such a diffusion mechanism is quite similar to the diffusion process in $\mathrm{CNNs}^{15}$.

Nearest-neighbor awareness naturally leads to a clustered network. The division of large networks into clusters consisting of cooperating nodes has several advantages such as increased robustness and security; simplified addressing, routing, and localization; lower memory requirements ${ }^{16,17}$ and lower energy consumption by enabling cooperative communication strategies ${ }^{18,19}$.

\subsection{Quality of service}

Quality of service refers to the capability of a network to deliver data reliably and timely. A high quantity of service, i.e., throughput or transport capacity (a distance-weighted sum-capacity), is generally not sufficient to satisfy an application's delay requirements. Consequently, the speed of propagation of information may be as crucial as the throughput.

The next Section will show how some of these issues can be addressed from a CNN perspective.

\section{The CNN Perspective}

\subsection{The traditional $C N N$ and extensions}

When the cellular neural (or nonlinear) network was first introduced in $1988^{20,21}$, the focus was on the class of single-layer, spatially invariant cellular neural networks (CNNs) defined on regular lattices. The dynamics of these networks is governed by a system 
of $n=M N$ differential equations,

$$
\begin{aligned}
\frac{d x_{i j}(t)}{d t}= & -x_{i j}(t)+\sum_{k, l \in \mathcal{N}_{i j}} a_{k-i, l-j} f\left(x_{k l}(t)\right) \\
& +\sum_{k, l \in \mathcal{N}_{i j}} b_{k-i, l-j} u_{k l}+I,
\end{aligned}
$$

where $x_{i j}$ denotes the states of a cell $(i, j) \in$ $\{1, \ldots, M\} \times\{1, \ldots, N\}$, and $\mathcal{N}_{i j}$ denotes the neighborhood of the cell at position $(i, j), a_{k l}$ the linear feedback template parameters (interconnection weights), $b_{k l}$ the linear feedforward template parameters, $u_{k l}$ the (time-invariant) input, and $I$ is a (spatially invariant) bias term. $f(\cdot)$ is the piecewise linear saturation function

$$
f(x)=\frac{1}{2}(|x+1|-|x-1|) .
$$

Spatial invariance of the connection weights is assumed; therefore, for nearest neighbor communication (8 neighbors), the template set $\mathbf{A}=\left\{a_{k l}\right\}$ and $\mathbf{B}=\left\{b_{k l}\right\}$ are $3 \times 3$ matrices. This type of CNN can be viewed at as a generalization of a cellular automaton; it constitutes a distributed nonlinear dynamical system with powerful modeling capabilities.

For the implementation of CNNs as sampled-data systems $^{22}$, discrete-time CNNs (DT-CNNs) were introduced $^{23}$. Their basic dynamic equation is

$$
\begin{aligned}
x_{i j}[t+1]= & \sum_{k, l \in \mathcal{N}_{i j}}\left(a_{k-i, l-j} f\left(x_{k l}[t]\right)+b_{k-i, l-j} u_{k l}[t]\right) \\
& +I .
\end{aligned}
$$

Note that in the original definition, the DT-CNN was restricted to the hardlimiting $\operatorname{sgn}(\cdot)$ nonlinearity.

The definition of CNNs was later extended to include all ensembles of cells that interact locally ${ }^{24,25,26,27}$, and cells with enhanced functionality were investigated ${ }^{28,29,30}$. The restriction to timeinvariant inputs and spatially invariant bias terms were lifted as well, and nonlinear templates have been investigated as early as $1992^{31,24}$ and implemented in hardware in $2000^{32}$.

\subsection{CNN models for DSNs}

In view of the characteristics of CNNs, it is apparent that DSNs are a subclass of CNNs, and it is expected that the analysis and design of DSNs are likely to benefit from the vast number of theoretical results CNNs.
The nodes or neurons of the DSN can be modeled as locally connected finite state machines, where the state may be binary (denoting, e.g., whether a node has a certain information or not), multi-valued, continuous, or a combination thereof. The state of a cell at time $k+1$ will depend on the states of the neighboring cells and sensor inputs at time $k$, which is precisely what (3) expresses. Since all the nodes run the same program, the rules for the state transitions will be identical, which means that the system is spatially invariant.

Using this CNN framework, important DSN problems (see Section 2) can be addressed. Propagation speed ${ }^{15,33}$, stability issues ${ }^{34,35,36}$, self-organization ${ }^{37}$, and dynamics of pattern formation $^{38,39}$ are well-studied areas in CNN theory, in which the understanding, analysis, and design of DSNs will certainly benefit.

Regular lattice models. In applications where the user has control over the node placement, nodes will often be arranged in rectangular lattice networks and communicate with four or eight nearest neighbors. This is the standard CNN model and, for communication networks, has been studied in numerous papers $^{40,41,42,43}$. The Manhattan network ${ }^{41,42}$ addresses the problem of boundary conditions, since it assumes a toroidal topology. While for DSNs, the impact of boundary conditions has been largely ignored, it is a well-studied subject in the CNN $\operatorname{area}^{44,38,45}$.

Probabilistic CNNs. Although deterministic "disk models" for the transmission range of a wireless node (see Fig. 1) have been used extensively and with some success for the analysis of sensor and ad hoc networks ${ }^{40,46,47,48,49}$, the volatility of the channel cannot be ignored in wireless networks ${ }^{10,7}$; the inaccuracy of "disk models" has also been pointed out in ${ }^{50}$ and is easily demonstrated experimentally ${ }^{51}$.

The stochastic nature of the wireless $\operatorname{link}^{52,53,54,55}$ can be modeled by a probabilistic $C N N$, in which each link is associated with a probability $p_{k l}$, in addition to the connection weights $a_{k l}$ and $b_{k l}$. The probability template $\mathbf{P}$ is of the same dimension as $\mathbf{A}$ and $\mathbf{B}$ and has entries $0 \leqslant p_{k l} \leqslant 1$ that are determined by the transmit power, path loss, noise, interference, fading, and the modulation, coding and 
receiver techniques. The probabilistic CNN equation can then be written as

$$
\begin{aligned}
x_{i j}[t+1]= & \sum_{k, l \in \mathcal{N}_{i j}}\left(a_{k-i, l-j} f\left(x_{k l}[t]\right)\right. \\
& \left.+b_{k-i, l-j} u_{k l}[t]\right) q_{k-i, l-j}[t],
\end{aligned}
$$

where $\mathbf{Q}[\cdot]=\left\{q_{k l}[\cdot]\right\}$ is a matrix random process with $q_{k l}[t]=1$ with probability $p_{k l}$ and $q_{k l}[t]=0$ with probability $1-p_{k l}$. With this framework, propagation and flooding can be studied, with special emphasis on networks with node failures, as faulty nodes are relatively common in sensor networks. While spatial invariance can be assumed for regular topologies, the $\mathbf{P}$ template will become spatially variant for other node distributions; however, it is still possible to relate reception probabilities to node distances.

\subsection{Other opportunities for CNN models}

It has been shown that CNNs can contribute to the routing problem, see the work on $\mathrm{CNN}$ path planning for a mobile robot in an environment with obstacles $^{56}$ and general path finding ${ }^{57,58}$.

CNN models have also been used to solve problems arising in communication networks such as packet switching 59,60 , and routing or path finding ${ }^{57,58}$.

Another promising application in the realm of $\mathrm{DSN}_{\mathrm{S}}$ is the use of CNNs for multisensor fusion ${ }^{61}$.

\section{Sensor Network Technology}

In this Section, we will introduce the technology behind sensor networks and show that not only CNN theory but also CNN hardware has potential applications in DSNs.

\subsection{The Berkeley "Motes"}

The Smart Dust project at the University of California at Berkeley ${ }^{62,63,64}$ aims at exploring the limits of system miniaturization by packing an autonomous sensing, computing, and communication node into a so-called "mote" with a volume of one cubic millimeter. This ambitious project requires both evolutionary and revolutionary advances in miniaturization, integration, and energy management. Such advances will be facilitated by the progress in MEMS, which permits the fabrication of small sensors, optical communication components, actuators, and power supplies. Microelectronics provides the necessary functionality and processing power in ever smaller areas with decreasing power and energy consumption. The Berkeley group follows two approaches, one is based on RF communication, the other on optical links.

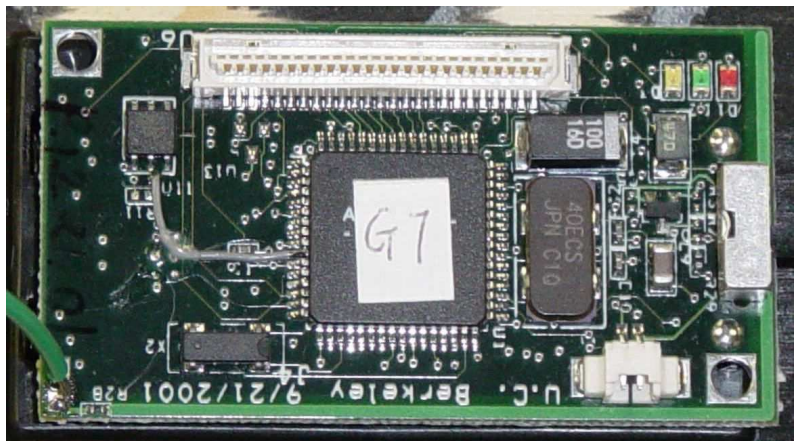

Fig. 2. The "Berkeley mote" RF wireless node (actual size).

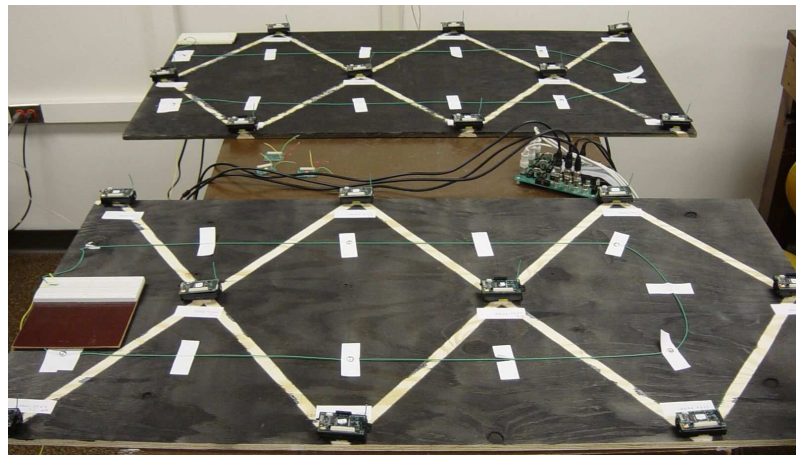

Fig. 3. A small regular network of motes.

RF motes. The current state of the RF mote (Figs. 2 and 3) is a matchbox sized PCB with off-theshelf components: A $4 \mathrm{MHz} 8$ bit CPU (by Atmel), a $50 \mathrm{kbit} / \mathrm{s}$ transceiver in the $900 \mathrm{MHz}$ band (by $\mathrm{RF}$ Monolithics), 4KB data memory, $128 \mathrm{~KB}$ program memory, and $6 \mathrm{~A} / \mathrm{D}$ channels to convert the sensor data. Various types of sensors such as accelerometers and magnetic, temperature, light, humidity, acoustic and pressure sensors can be connected via a generic interface. An extremely compact operating system called "TinyOS" has been tailor-made for this type of mote ${ }^{65}$.

Of course this mote is far from fitting in a cubic millimeter, but merely by combining its components into a single chip, its volume could be decreased by two orders of magnitude. 
Optical motes. As discussed in the Section on power supply, a promising alternative to $\mathrm{RF}$ communication are laser-based optical links. The main disadvantage is that a line-of-sight path has to be available, and the main challenge is to aim the laser beam precisely at the receiving mote. Additionally, the laser diodes are relatively power-hungry. The Berkeley group managed to come up with an elegant solutions to these problems by implementing a passive transmission technique ${ }^{62}$, i.e., to modulate incoming optical signals and reflect them. This idea is based on a corner-cube retroreflector (CCR) that comprises three mutually perpendicular mirrors of gold-coated polysilicon. The CCR has the property that any incident ray of light is reflected back to its source. The MEMS-based CCR includes an electrostatic actuator that can deflect one of the mirrors at kilohertz rates. It has been demonstrated that a CCR illuminated by an external light source can transmit back a modulated signal at kilobits per second. For the active laser transmitter, MEMS technology can be used to assemble a beam-steering micro-mirror to enable communication between nodes. For passive transmission, it is assumed that the operator of the DSN is using a relatively high power laser beam to "interrogate" the motes. Such an interrogating beam could be built in binoculars, for example. For the receiver, a CCD camera is suggested, that will have to detect numerous incoming signals simultaneously.

For an envisioned size of up to $256 \times 256$ pixels, this CCD array will generate a data flow of several tens of Mbits/s, which cannot be handled in a hand-held device. Clearly, the incoming data carried by the modulated reflected laser beams has to preprocessed and/or compressed early, most beneficially directly at the CCD sensor. Such a task is certainly tailored to CNNs with optical inputs ${ }^{66,67,68,69}$, since the CNN processing capability permits an efficient detection of relevant patterns in the motes' response.

\subsection{Combining sensors and actuators}

The Berkeley platform includes mirrors as actuators in the optical mote, but that does not enable it to react to the environment. Numerous applications only become attractive if the nodes are mobile and are able to react to what they see or what the network as a whole sees. Mobile platforms could in- clude small mobile robots or flying insect-like nodes. Such an extension necessarily entails closed sensoractuator control loops. Since sensor and actuator are generally not colocated on one node, the control problem is distributed, and it suffers from unknown delays induced by the underlying network. Closedloop control aspects of large networks are the subject of a research effort sponsored by DARPA/IXO* This work is currently in progress; the goal is the formal verification of the correctness of the distributed control algorithm, and to derive tight upper bounds on the delay of the feedback loops. As with CNNs, such feedback leads to much richer dynamics but necessitates measures to guarantee stability.

\section{An example of DSN-CNN modeling: Syn- chronization}

Preliminary studies have demonstrated the potential of the DSN-CNN to model clustering phenomena, in particular for synchronization algorithms. In recent hardware experiments at Notre Dame on a testbed with 50 motes (see Fig. 4) using a modelbased algorithm ${ }^{70}$, different clusters of tightly synchronized nodes could be identified, with a relatively high inter-cluster synchronization error. These observations can be modeled and explained using CNNs.

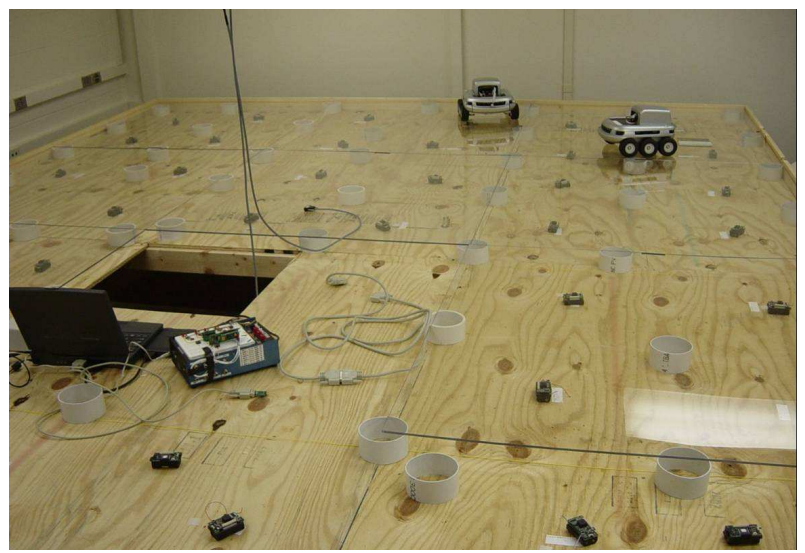

Fig. 4. Testbed with 50 motes in a regular grid network. The robots are present to generate events that the sensor can detect.

It turned out that clusters emerged with tight intra-cluster synchronization but loose inter-cluster synchronization. Such a phenomenon can easily be 
explained with the DSN-CNN model: The topology of the network is a regular lattice, and we assume communication (i.e., exchange of clock information) to the eight nearest neighbors. The state of a CNN node represents the (relative) state of the clock of the corresponding DSN node. Clearly, for a binary CNN, at most two (stable) states are possible. This has been sufficient to model the dynamics that we observed in experiments with comparatively small networks.

Since the four diagonal neighbors are further away than the four other ones, the connection is weaker. DSNs normally use simple isotropic (in the plane) radiators as antennas, thus template parameters reflecting the wireless channel are symmetric. The path loss can be modeled as follows: $a_{i j}, b_{i j} \propto$ $\left(i^{2}+j^{2}\right)^{-\alpha / 2}$, where $\alpha$ is the path loss exponent, signifying that the received signal power over a distance $d$ from the transmitter is proportional to $d^{-\alpha}$. There is no self-feedback in the synchronization algorithm, and the exchange of packets is usually symmetric. These properties are subsumed in the following symmetric template:

$$
A=\left[\begin{array}{ccc}
0.125 & 0.25 & 0125 \\
0.25 & 0 & 0.25 \\
0.125 & 0.25 & 0.125
\end{array}\right] ; \quad B=[0] ; \quad I=0 .
$$

In this case, since the distance to the diagonal neighbors is $\sqrt{2}$ times the distance to the nearest neighbors, the template parameters correspond to a path loss exponent of 2 (line-of-sight propagation).

When initialized with small random variations around zero, corresponding to initial deviations of the clocks, the network forms clusters relatively quickly (Fig.5). Eventually, after a long phase of boundary negotiation, one of the states dominates, and the networks ends up tightly synchronized.

Since the feedback template is symmetric, the DSN-CNN is stable, which suggests that the synchronization algorithm will eventually converge to an equilibrium state. If the symmetry is broken, the clusters move across the network, which can be explained by the fact that in this case, some nodes impose more clock information on their neighbors than vice versa. In this case, an equilibrium may never be achieved.

All these dynamics can be verified on the CNN simulator available at http://www.isi.ee.ethz. $\mathrm{ch} /{ }^{\sim}$ haenggi/CNNsim_adv.html ${ }^{71}$.

Unfortunately, due to practical limits, we are unable to run experiments with more than 50 nodes. It would be interesting to see whether for larger networks, more clusters with different synchronization states emerge. Such a behavior could be modeled by a multi-state $\mathrm{CNN}$.

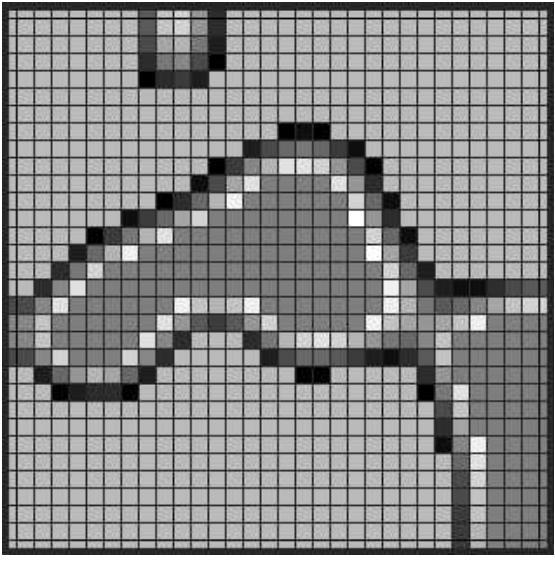

Fig. 5. Snapshot of the synchronization dynamics in a $30 \times 30$ DSN-CNN model. Clusters have formed, and the boundary negotiation is in progress.

\section{Concluding Remarks}

The main goal of this article was the introduction of DSNs to the neural systems community as an emerging technological platform that may greatly benefit from the rich theory that is available for CNNs and other neural systems.

DSNs are very promising systems for pervasive sensing, surveillance, and computing which may revolutionize information gathering and processing. As miniaturization proceeds further, goals such as cubic millimeter "smart dust" become feasible.

Networking with such tiny nodes is closely related to the concept of nanonetworking that we recently introduced $^{72}$, which denotes networking with nodes that are based on nanoelectronic devices. CNNs based on resonant tunneling diodes ${ }^{73}$ or quantum $\operatorname{dots}^{74,75,76}$ are the first examples of such nanonets. The wireless nanonets we envision have the potential to surpass the barrier of $1 \mathrm{~mm}^{3}$ per node, thus coming even closer to real smart dust.

In summary, we note that there is an unconsummated union between nonlinear dynamical systems such as the CNN and distributed sensor networks. Clearly, DSNs are related to CNNs in multiple ways. 
Not only the topology with its nearest-neighbor connectivity is similar, but also the process how information is diffused or propagated through the network. The two main strengths of the CNN paradigm are expected to have an impact on DSN technology: $C N N$ theory, applied to a probabilistic $\mathrm{CNN}$, is expected to enable the exploration of the dynamics and behavior of DSNs; CNN hardware may become significant for processing data gathered in the interrogating device of a passive optically connected network.

Since this is a new and interdisciplinary perspective of DSNs, we compiled an extensive list of references in both the CNN and the DSN areas for the interested reader.

\section{Acknowledgment}

The partial support of the DARPA/IXO-NEST Program (contract AF-F30602-01-2-0526) is gratefully acknowledged.

\section{References}

1. G. J. Pottie and W. J. Kaiser, "Wireless integrated network sensors," Communications of the ACM, vol. 43, no. 5, pp. 551-558, 2000.

2. D. Estrin, L. Girod, G. Pottie, and M. Srivastava, "Instrumenting the world with wireless sensor networks," in International Conference on Acoustics, Speech \& Signal Processing, vol. 4, (Salt Lake City, UT), pp. 2033-2036, 2001.

3. I. F. Akyildiz, W. Su, Y. Sankarasubramaniam, and E. Cayirci, "Wireless sensor networks: a survey," Computer Networks, vol. 38, pp. 393-422, Mar. 2002.

4. D.L. Hall and J. Llinas (editors), Handbook of Multisensor Data Fusion. CRC Press, 2001.

5. C. E. Perkins, ed., Ad Hoc Networking. Addison Wesley, 2000. ISBN 0-201-30976-9.

6. M. Frodigh, P. Johansson, and P. Larsson, "Wireless ad hoc networking - The art of networking without a network," Ericsson Review, no. 4, pp. 248-263, 2000. Available at: http://www.ericsson.com/about/ publications/review/2000_04/article124.shtml.

7. A. J. Goldsmith and S. B. Wicker, "Design Challenges for Energy-Constrained Ad Hoc Wireless Networks," IEEE Wireless Communications, vol. 9, pp. 8-27, Aug. 2002.

8. Internet Engineering Task Force, "Mobile Ad-hoc Networks (MANET)." See http://www.ietf.org/ html. charters/manet-charter.html.

9. C.-K. Toh, Ad Hoc Mobile Wireless Networks - Protocols and Systems. Prentice-Hall, 2002. ISBN 0-13007817-4.
10. A. Ephremides, "Energy Concerns in Wireless Networks," IEEE Wireless Communications, vol. 9, pp. 48-59, Aug. 2002.

11. V. Rodoplu and T. H. Meng, "Minimum Energy Mobile Wireless Networks," IEEE Journal on Selected Areas in Communications, vol. 17, no. 8, pp. 13331344, 1999.

12. "Bluetooth Wireless Technology." Official Bluetooth site: http://www.bluetooth.com.

13. E. M. Royer and C.-K. Toh, "A Review of Current Routing Protocols for Ad-Hoc Mobile Wireless Networks," IEEE Personal Communications, vol. 6, pp. 46-55, Apr. 1999.

14. C. Intanagowiwat, R. Govindan, and D. Estrin, "Directed diffusion: A scalable and robust communication paradigm for sensor networks," in ACM International Conference on Mobile Computing and Networking (MobiCom), (Boston, MA), pp. 56-67, Aug. 2000.

15. P. Thiran, G. Setti, and M. Hasler, "An Approach to Information Propagation in 1-D Cellular Neural Networks - Part I: Local Diffusion," IEEE Transactions on Circuits and Systems-I, vol. 45, pp. 777-789, Aug. 1998.

16. I. Chlamtac and A. Farago, "A new approach to the design and analysis of peer-to-peer mobile networks," Wireless Networks, vol. 5, pp. 149-156, 1999.

17. A. B. McDonald and T. F. Znati, "Design and Performance of a Distributed Dynamic Clustering Algorithm for Ad-Hoc Networks," in Simulation Symposium, pp. 27-34, Aug. 2001.

18. M. Haenggi, "A Formalism for the Analysis and Design of Time and Path Diversity Schemes in Wireless Sensor Networks," in The 2nd International Workshop on Information Processing in Sensor Networks (IPSN'03), (Palo Alto, CA), pp. 417-431, Apr. 2003. Available at http://www.nd.edu/ mhaenggi/ ipsn03.pdf.

19. J. Laneman, D. Tse, and G. Wornell, "Cooperative Diversity in Wireless Networks: Efficient Protocols and Outage Behavior," IEEE Transactions on Information Theory. Submitted for publication. Available at: http://www.nd.edu/ jnl/pubs/it2002.pdf.

20. L. O. Chua and L. Yang, "Cellular Neural Networks: Theory," IEEE Transactions on Circuits and Systems-I, vol. 35, pp. 1257-1272, Oct. 1988.

21. L. O. Chua and L. Yang, "Cellular Neural Networks: Applications," IEEE Transactions on Circuits and Systems-I, vol. 35, pp. 1273-1290, Oct. 1988.

22. H. Harrer and J. A. Nossek, "An analog implementation of discrete-time CNNs," IEEE Transactions on Neural Networks, vol. 3, 1992.

23. H. Harrer and J. A. Nossek, "Discrete-time cellular neural networks," International Journal of Circuit Theory and Applications, vol. 20, Sept. 1992.

24. T. Roska and L. O. Chua, "Cellular Neural Networks with Non-Linear and Delay-Type Template Elements 
and Non-Uniform Grids," International Journal of Circuit Theory and Applications, vol. 20, pp. 469-481, September 1992.

25. L. O. Chua and T. Roska, "The CNN Paradigm," IEEE Transactions on Circuits and Systems-I, vol. 40, pp. 147-156, Mar. 1993.

26. L. O. Chua, "CNN: A Vision of Complexity," International Journal of Bifurcation and Chaos, vol. 7, pp. 2219-2425, Oct. 1997.

27. L. O. Chua, CNN: A Paradigm for Complexity. World Scientific, Singapore, 1998. ISBN 9-81-023483-X.

28. A. Kanagawa, H. Kawabata, and H. Takahashi, "Cellular neural networks with multiple-valued output and its application," IEICE Transactions on Fundamentals of Electronics, Communications and Computer Sciences, vol. E79-A, Oct. 1996.

29. R. Dogaru, L. O. Chua, and K. R. Crounse, "Pyramidal cells: A novel class of adaptive coupling cells and their applications for cellular neural networks," IEEE Transactions on Circuits and Systems-I, vol. 45, pp. 1077-1090, Oct. 1998.

30. R. Dogaru and L. O. Chua, "Universal CNN Cells," International Journal of Bifurcation and Chaos, vol. 9, pp. 1-48, Jan. 1999.

31. S. Jankowski and R. Wańczuk, "Nonlinear CNN Cloning Templates for Image Thicking," in IEEE International Workshop on Cellular Neural Networks and their Applications, (München), pp. 197-202, Oct. 1992.

32. A. Paasio and K. Halonen, "Cellular Nonlinear Network Implementation for Nonlinear B-Template," in IEEE International Workshop on Cellular Neural Networks and their Applications, (Catania, Italy), pp. 437-442, May 2000.

33. M. Hänggi and G. S. Moschytz, "An Analysis of CNN Settling Time," IEEE Transactions on Circuits and Systems-I, vol. 47, pp. 9-24, Jan. 2000.

34. L. O. Chua and T. Roska, "Stability of a Class of Nonreciprocal Cellular Neural Networks," IEEE Transactions on Circuits and Systems-I, vol. 37, pp. 15201527, Dec. 1990.

35. F. Zou and J. A. Nossek, "Stability of Cellular Neural Networks with Opposite-Sign Templates," IEEE Transactions on Circuits and Systems, vol. 38, pp. 675-677, June 1991.

36. T. Roska, C. W. Wu, and L. O. Chua, "Stability of Cellular Neural Networks with Dominant Nonlinear and Delay-type Templates," IEEE Transactions on Circuits and Systems-I, vol. 40, pp. 270-272, Apr. 1993.

37. P. Thiran, Dynamics and Self-organization of Locally Coupled Neural Networks. Collection META, Lausanne, Switzerland: Presses Polytechniques et Universitaires Romandes, 1997. ISBN 2-88074-351-6.

38. P. Thiran, K. R. Crounse, L. O. Chua, and M. Hasler, "Pattern Formation Properties of Autonomous Cellular Neural Networks," IEEE Transactions on Circuits and Systems-I, vol. 42, pp. 757-774, Oct. 1995.

39. K. R. Crounse, L. O. Chua, P. Thiran, and G. Setti, "Characterization and dynamics of pattern formation in cellular neural networks," International Journal of Bifurcation and Chaos, vol. 6, pp. 1703-1724, Sept. 1996.

40. J. A. Silvester and L. Kleinrock, "On the Capacity of Multihop Slotted ALOHA Networks with Regular Structure," IEEE Transactions on Communications, vol. COM-31, pp. 974-982, Aug. 1983.

41. N. F. Maxemchuck, "Routing in the Manhattan Street Network," IEEE Transactions on Communications, vol. COM-35, pp. 503-512, May 1987.

42. G. Mergen and L. Tong, "On the Capacity of Regular Wireless Networks with Transceiver Multipacket Communication," in IEEE International Symposium on Information Theory, (Lausanne, Switzerland), p. 350, 2002.

43. L.-L. Xie and P. R. Kumar, "A Network Information Theory for Wireless Communication: Scaling Laws and Optimal Operation," Apr. 2002. Submitted to IEEE Transactions on Information Theory. Available at: http://black1.csl.uiuc.edu/ prkumar/publications.htm.

44. P. Thiran, "Influence of the boundary conditions on the behavior of Cellular Neural Networks," IEEE Transactions on Circuits and Systems-I, vol. 40, pp. 207-212, 1993.

45. M. Hänggi and G. S. Moschytz, Cellular Neural Networks: Analysis, Design and Optimization. Kluwer Academic Publishers, 2000. ISBN 0-7923-7891-1.

46. H. Takagi and L. Kleinrock, "Optimal Transmission Ranges for Randomly Distributed Packet Radio Terminals," IEEE Transactions on Communications, vol. COM-32, pp. 246-257, Mar. 1984.

47. L. Hu, "Topology Control for Multihop Packet Networks," IEEE Transactions on Communications, vol. 41, no. 10, pp. 1474-1481, 1993.

48. J. L. Wang and J. A. Silvester, "Maximum Number of Independent Paths and Radio Connectivity," IEEE Transactions on Communications, vol. 41, pp. 14821493, Oct. 1993.

49. P. Gupta and P. R. Kumar, "The Capacity of Wireless Networks," IEEE Transactions on Information Theory, vol. 46, pp. 388-404, Mar. 2000.

50. E. S. Sousa and J. A. Silvester, "Optimum Transmission Ranges in a Direct-Sequence Spread-Spectrum Multihop Packet Radio Network," IEEE Journal on Selected Areas in Communications, vol. 8, pp. 762771, June 1990.

51. D. A. Maltz, J. Broch, and D. B. Johnson, "Lessons from a Full-Scale Multihop Wireless Ad Hoc Network Testbed," IEEE Personal Communications, vol. 8, pp. 8-15, Feb. 2001.

52. M. Zorzi and S. Pupolin, "Optimum Transmission Ranges in Multihop Packet Radio Networks in the Presence of Fading," IEEE Transactions on Commu- 
nications, vol. 43, pp. 2201-2205, July 1995.

53. Y. Y. Kim and S. Li, "Modeling Multipath Fading Channel Dynamics for Packet Data Performance Analysis," Wireless Networks, vol. 6, pp. 481-492, 2000

54. T. S. Rappaport, Wireless Communications - Principles and Practice. Prentice Hall, 2nd ed., 2002. ISBN 0-13-042232-0.

55. M. Haenggi, "Probabilistic Analysis of a Simple MAC Scheme for Ad Hoc Wireless Networks," in IEEE CAS Workshop on Wireless Communications and Networking, (Pasadena, CA), Sept. 2002.

56. A. Gacsadi, T. Maghlar, and V. Tiponut, "A CNN path planning for a mobile robot in an environment with obstacles," in IEEE International Workshop on Cellular Neural Networks and their Applications, (Frankfurt, Germany), pp. 188-194, July 2002.

57. H. Kim, Y. Park, T. Roska, and L. Chua, "Optimal path finding with space variant metric weights via multilayer CNN-UM," in IEEE International Symposium on Circuits and Systems, vol. 2, (Sydney, Australia), pp. 429-432, May 2001.

58. H. Kim, T. Roska, L. Chua, and F. Werblin, "Initiation and tracking of dim target via fusion of feature probabilities with CNN-UM," in IEEE International Symposium on Circuits and Systems, vol. 1, (Phoenix, AZ), pp. 225-228, May 2002.

59. R. Fantacci, M. Forti, and M. Marini, "A Cellular Neural Network for Packet Selection in a Fast Packet Switching Fabric with Input Buffers," IEEE Transactions on Communications, pp. 1649-1652, Dec. 1996.

60. R. Fantacci, M. Forti, M. Marini, and L. Pancani, "Cellular Neural Network Approach to a Class of Communication Problems," IEEE Transactions on Circuits and Systems-I, pp. 1457-1467, Dec. 1999.

61. P. Arena, S. Baglio, L. Fortuna, and S. Graziani, "Analog Cellular Networks for Multisensor Fusion and Control," IEEE Transactions on Circuits and Systems-I, vol. 47, pp. 1378-1382, Sept. 2000.

62. J. M. Kahn, R. H. Katz, and K. S. Pister, "Emerging challenges: Mobile networking for 'smart dust'," Journal of Communications and Networks, vol. 2, Sept. 2000.

63. B. Atwood, B. Warneke, and K. Pister, "Preliminary Circuits for Smart Dust," in Southwest Symposium on Mixed-Signal Design, (San Diego, CA), pp. 87-92, Feb. 2000.

64. B. Warneke, B. Atwood, and K. Pister, "Smart dust mote forerunners," in 14th IEEE International Conference on MEMS, pp. 357-360, 2001.

65. J. Hill, R. Szewczyk, A. Woo, D. Culler, S. Hollar, and K. Pister, "System Architecture Directions for Networked Sensors," in International Conference on Architectural Support for Programming Languages and Operating Systems (ASPLOS), (Cambridge, MA), Nov. 2000.
66. S. Espejo, R. Domínguez-Castro, and A. RodríguezVázquez, "A Realization of a CNN Universal Chip in CMOS Technology," in IEEE International Symposium on Circuits and Systems, vol. 3, (Seattle), pp. 657-659, May 1995.

67. R. Domínguez-Castro, S. Espejo, A. RodríguezVázquez, R. Carmona, P. Földesy, Á. Zarándy, P. Szolgay, T. Szirányi, and T. Roska, "A 0.8um CMOS Two-Dimensional programmable mixed-signal focal-plane array processor which on-chip binary imaging and instruction storage," IEEE Journal of Solid State Circuits, vol. 32, pp. 1013-1026, July 1997.

68. A. Rodríguez-Vázquez, S. Espejo, R. DomínguezCastro, and G. Liñán, "The $64 \times 64$ analog input CNN Universal Machine Chip and its ARAM," in International Symposium on Nonlinear Theory and its Applications, vol. 2, (Crans-Montana, Switzerland), pp. 667-670, Sept. 1998.

69. G. Liñan, S. Espejo, R. Domínquez-Castro, and A. Rodríguez-Vázquez, " $64 \times 64 \mathrm{CNN}$ universal chip with analog and digital I/O," in Proceedings of the IEEE International Conference on Electronics, Circuits and Systems, (Lisbon), pp. 203-206, Sept. 1998.

70. M. Lemmon, J. Ganguly, and L. Xia, "Modelbased clock synchronization in networks with drifting clocks," in Pacific Rim International Symposium on Dependable Computing, pp. 177-184, 2000.

71. M. Hänggi, S. Moser, E. Pfaffhauser, and G. S. Moschytz, "Simulation and Visualization of CNN Dynamics," International Journal of Bifurcation and Chaos, vol. 9, pp. 1237-1261, July 1999.

72. M. Haenggi, "From Nanodevices to Distributed Systems: Nanonetworks," Nov. 2002. Invited talk in the IEEE CAS Tour on Nanotechnology and Gigascale Systems (Bahia Blanca, Argentina, and Puebla, Mexico). Available at http://www.nd.edu/ mhaenggi/ castour.pdf.

73. M. Hänggi and L. O. Chua, "Cellular Neural Networks Based on Resonant Tunneling Diodes," International Journal of Circuit Theory and Applications, vol. 29, pp. 487-504, Sep./Oct. 2001.

74. G. Toth, C. Lent, P. Tougaw, Y. Brazhnik, W. Weng, W. Porod, R.-W. Liu, and Y.-F. Huang, "Quantum cellular neural networks," Superlattices and Microstructures, vol. 20, no. 4, 1996.

75. W. Porod, "Towards Nanoelectronics: Possible CNN Implementations using Nanoelectronic Devices," in IEEE International Workshop on Cellular Neural Networks and their Applications, (London), pp. 2025, 1998.

76. C. Gerousis, S. M. Goodnick, and W. Porod, "Toward nanoelectronic cellular neural networks," International Journal of Circuit Theory and Applications, vol. 28, pp. 523-535, Nov/Dec. 2000. Special issue on nanoelectronic circuits. 